Check for updates

Cite this: Chem. Sci., 2017, 8, 4853

\title{
Fluoride-promoted carbonylation polymerization: a facile step-growth technique to polycarbonates $\uparrow$
}

\begin{abstract}
J. V. Olsson, \$ D. Hult, (D) \& S. García-Gallego (D) and M. Malkoch (D)*
Fluoride-Promoted Carbonylation (FPC) polymerization is herein presented as a novel catalytic polymerization methodology that complements ROP and unlocks a greater synthetic window to advanced polycarbonates. The overall two-step strategy is facile, robust and capitalizes on the synthesis and step-growth polymerization of bis-carbonylimidazolide and diol monomers of 1,3- or higher configurations. Cesium fluoride (CsF) is identified as an efficient catalyst and the bis-carbonylimidazolide monomers are synthesized as bench-stable white solids, easily obtained on 50-100 g scales from their parent diols using cheap commercial 1,1'-carbonyldiimidazole (CDI) as activating reagent. The FPC polymerization works well in both solution and bulk, does not require any stoichiometric additives or complex settings and produces only imidazole as a relatively low-toxicity by-product. As a proof-ofconcept using only four diol building-blocks, FPC methodology enabled the synthesis of a unique library of polycarbonates covering (i) rigid, flexible and reactive PC backbones, (ii) molecular weights 5-20 kg $\mathrm{mol}^{-1}$, (iii) dispersities of 1.3-2.9 and (iv) a wide span of glass transition temperatures, from -45 up to $169^{\circ} \mathrm{C}$.
\end{abstract}

Received 21st December 2016

Accepted 3rd May 2017

DOI: $10.1039 / c 6 s c 05582 f$

rsc.li/chemical-science synthetic approaches have successfully been demonstrated by Hedrick and coworkers ${ }^{8}$ as well as by our group. ${ }^{9}$ Another approach, entailing a scalable ring-closing depolymerization, has recently been demonstrated by Olsén et al. ${ }^{\mathbf{1 0}}$ Nevertheless, monomer purification and stability issues involving autopolymerization remain challenging making ROP a less attractive upscaling technique to produce APCs. It should also be noted that because of thermodynamic ring-strain parameters of cyclic carbonates, ROP processes are mostly restricted to 1,2 and 1,3-diol precursors.

On the other hand, traditional step-growth polymerization protocols involving phosgene or dialkyl carbonates are costeffective, scalable and can be utilized for polycarbonate production from a broader selection of bulk diols including BPA., Unfortunately, for densely functionalized polycarbonates, these techniques are still considered as inappropriate mainly because of harsh reaction conditions including high temperatures and/or concomitant formation of corrosive/toxic by-products. ${ }^{1}$ For example, $\mathrm{HCl}$ is generated from phosgene which requires stoichiometric base for neutralization and toxic phenol is obtained from polymerization with diphenyl carbonate (DPC). In addition, the reversibility of the condensation polymerization process with dialkyl carbonates, demands forcing reaction conditions involving high temperature and vacuum to ensure continuous removal of the alcoholic/phenolic by-products. Consequently, careful control of temperature and pressure is necessary, rendering these methods operationally challenging and often impractical for standard laboratory scale synthesis. In comparison, alternating copolymerization of epoxides with carbon dioxide has emerged as
Department of Coating Technology, Division of Fibre and Polymer Technology, KTH Royal Institute of Technology, Teknikringen 56-58, 100 44, Stockholm, Sweden. E-mail: malkoch@kth.se

$\dagger$ Electronic supplementary information (ESI) available: Experimental details for the synthesis and structural characterization of monomers and polymers, and kinetic study. See DOI: 10.1039/c6sc05582f

\$ These authors contributed equally to this paper. 


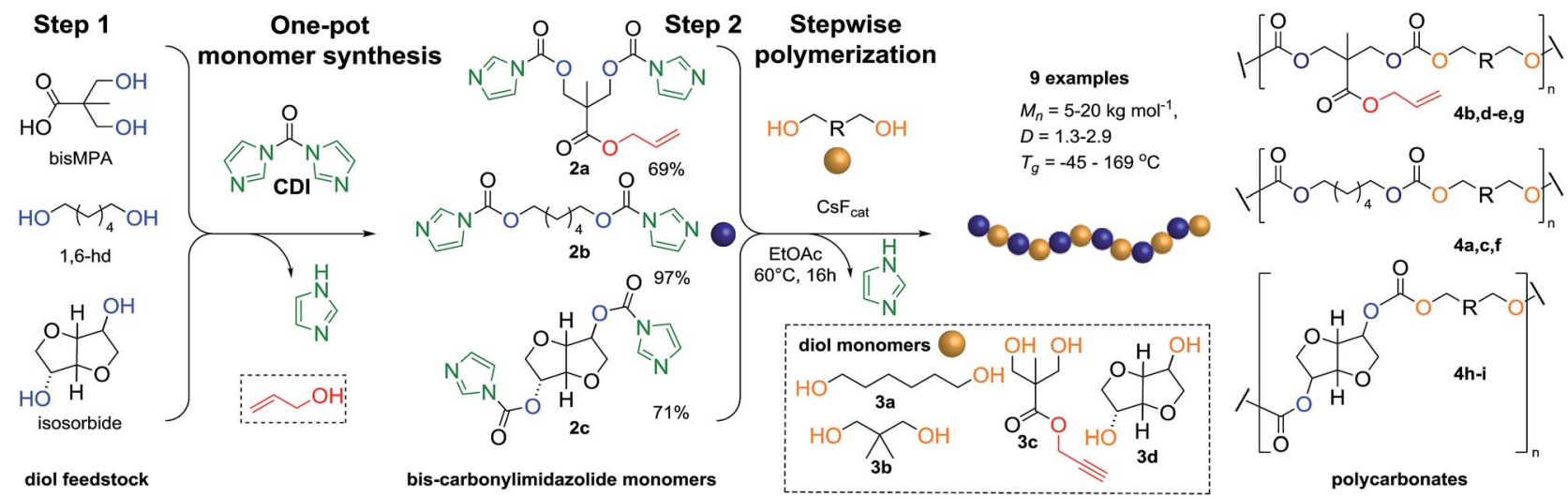

Scheme 1 Overall two step strategy of FPC. Step 1: carbonylimidazolide activation of monomers and step 2: step-growth polymerization into APCs via FPC polymerization.

a versatile polymerization technique with high atom-efficiency albeit still limited by poor availability of functional epoxides. ${ }^{5,11,12}$ The absence of practical and high-fidelity synthetic strategies that unlock a greater number of functional polycarbonates has most likely inhibited their broader use in application-driven research.

The ever-increasing need for advanced materials prompted us to investigate a new synthetic approach with emphasis on operational practicality and functional group tolerance, similar to ROP protocols, and in parallel offering a broad substrate scope and scalability of traditional step-growth polymerization processes. In this context, we herein disclose a novel two-step synthetic strategy that expands the window of functional polycarbonates from simple bis-carbonylimidazolide and diol building blocks via step-growth using CsF as an innovative polymerization catalyst (Scheme 1).

\section{Results and discussion}

The FPC technique was found versatile for generating polycarbonates from 1,3- and higher diols also bearing pendant functional groups. In the first step, as a proof-of-concept, three diols were transformed to bis-carbonylimidazolide activated monomers $\mathbf{2 a - c}$. These were carefully selected to cover properties such as (i) functionality from the orthogonal 2,2-bis(hydroxymethyl)propionic acid (bis-MPA) 2a; (ii) flexibility of 1,6hexanediol 2b; (iii) and rigidity of isosorbide $2 \mathbf{c}$. All three biscarbonylimidazolides $\mathbf{2 a - c}$ could be isolated as bench-stable white solids on large synthetic scales by reaction with $1,1^{\prime}$-carbonyldiimidazole (CDI). For instance, monomer 2a was obtained on $92 \mathrm{~g}$ scale (69\% overall yield) directly from bis-MPA by an operationally simple one-pot synthetic protocol using 3 equivalents of CDI, followed by a chemoselective in situ reaction with allyl alcohol. In contrast to its cyclic carbonate analogue, ${ }^{9}$ purification of bis-carbonylimidazolide $\mathbf{2 a}$ was accomplished by removing excess imidazole through simple acidic extraction and washing with diethyl ether. The structure and purity of 2a-c were confirmed by ${ }^{1} \mathrm{H}$ - and ${ }^{13} \mathrm{C}-\mathrm{NMR}$ analysis (Fig. S2-S4†).

CDI has previously been applied as a versatile reagent in organic synthesis from peptides,$^{13}$ glycosidation, ${ }^{14}$ assymetric and 1,2-cyclic carbonates, ${ }^{15}$ urethane ${ }^{16,17}$ and carbonate ${ }^{18}$ dendrimers, to large scale manufacturing. ${ }^{19}$ Surprisingly, the use of bis-carbonylimidazolides as monomers for generating polycarbonates has been a largely unexplored research ground, despite the obvious advantages including easily available monomers and the relative low toxicity of the imidazole byproduct. One of the few reported examples so far was published by Fréchet and co-workers in $1985,{ }^{20}$ requiring a combination of stoichiometric base along with a crown ether catalyst.
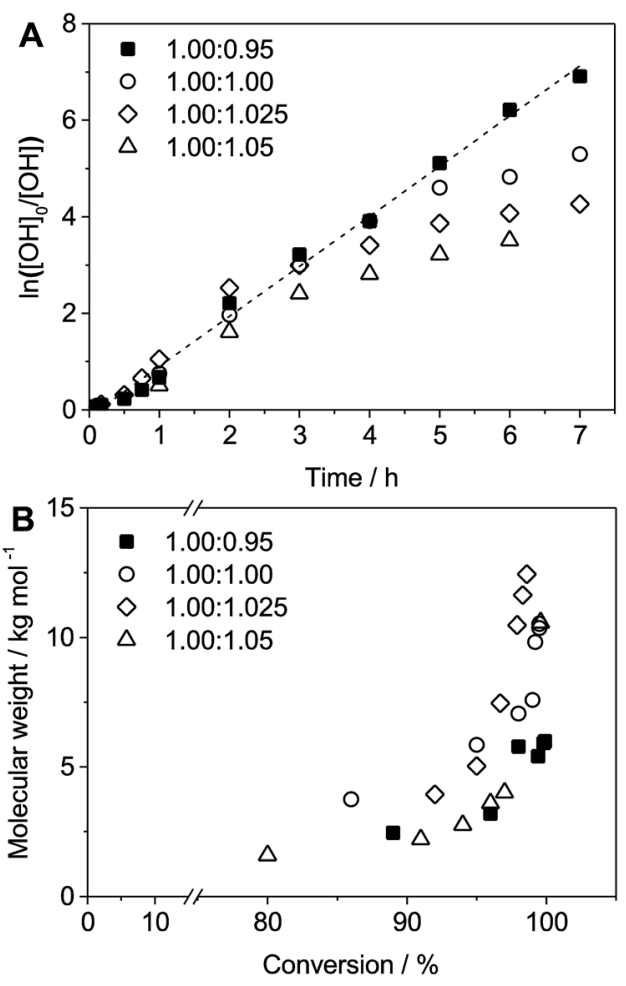

Fig. 1 (A) Kinetic plot of $\ln \left([\mathrm{OH}]_{0} /[\mathrm{OH}]\right)$ vs. time, for step-growth polymerization of 1,6-hexanediol $3 a$ and $2 b$ in four different ratios using $5 \mathrm{~mol} \% \mathrm{CsF}$ in EtOAc, as obtained from ${ }^{1} \mathrm{H}-\mathrm{NMR}$. Dashed line represent linear fit to ( $\mathbf{\square}), R^{2}=0.99$. (B) Plots of $M_{\mathrm{n}}$ vs. monomer conversion, as obtained from ${ }^{1} \mathrm{H}-\mathrm{NMR}$ and SEC. 
Considering the sustainability challenges ahead, it is apparent that APCs need to be produced under more benign conditions which circumvent the need for stoichiometric additives as well as proceed under mild reaction conditions. ${ }^{21} \mathrm{We}$ identified CsF as a soft and efficient catalyst that can overcome these challenges. Due to CsF's selective mode of activation and high fidelity for alcohol-induced displacement of imidazolideactivated carboxylated monomers, fluoride-promoted esterification was recently demonstrated as a robust, efficient and sustainable process to generate monodisperse polyester dendrimers under green chemistry conditions. ${ }^{22}$

In order to prove the hypothesis of Fluoride-Promoted Carbonylation (FPC) polymerization as a suitable strategy towards APCs, an initial screening of reaction conditions was performed using diol $\mathbf{3 a}$ and bis-carbonylimidazolides $\mathbf{2 a}$ (Scheme S2, Table $\mathbf{S} 2 \dagger$ ) and $\mathbf{2 b}$ (Scheme S1, Table S1 $\dagger$ ). Indeed, the efficiency and robustness of CsF as catalyst could be confirmed using a catalytic loading of $5 \mathrm{~mol} \%$ in ethyl acetate, resulting in full diol monomer conversion after 16 hours at $65{ }^{\circ} \mathrm{C}$. Notably, the reaction was more effective in bulk using lower catalytic loadings ( $1 \mathrm{~mol} \%$ ) at elevated temperature (90$100{ }^{\circ} \mathrm{C}$ ) for 3 hours. A slight offset diol : bis-carbonylimidazolide ratio of $1.00: 1.05$ was employed to counteract the possible in situ hydrolysis caused by non-dried solvents, and proved optimal as it worked for both bis-carbonylimidazolides 2a and 2b in generating APCs with $M_{\mathrm{n}}$ between 9 and $16 \mathrm{~kg} \mathrm{~mol}^{-1}$.

Kinetic studies of polymerization runs with different stoichiometric ratios using diol $\mathbf{3 a}$ and bis-carbonyldiimidazolide 2b were monitored by ${ }^{1} \mathrm{H}-\mathrm{NMR}$ and Size Exclusion Chromatography (SEC). The results, depicted in Fig. 1, support a traditional step-growth polymerization mechanism, displaying exponential molecular weight increase at high monomer conversion (Fig. 1B).

Table 1 FPC of bis-carbonylimidazolides and diols

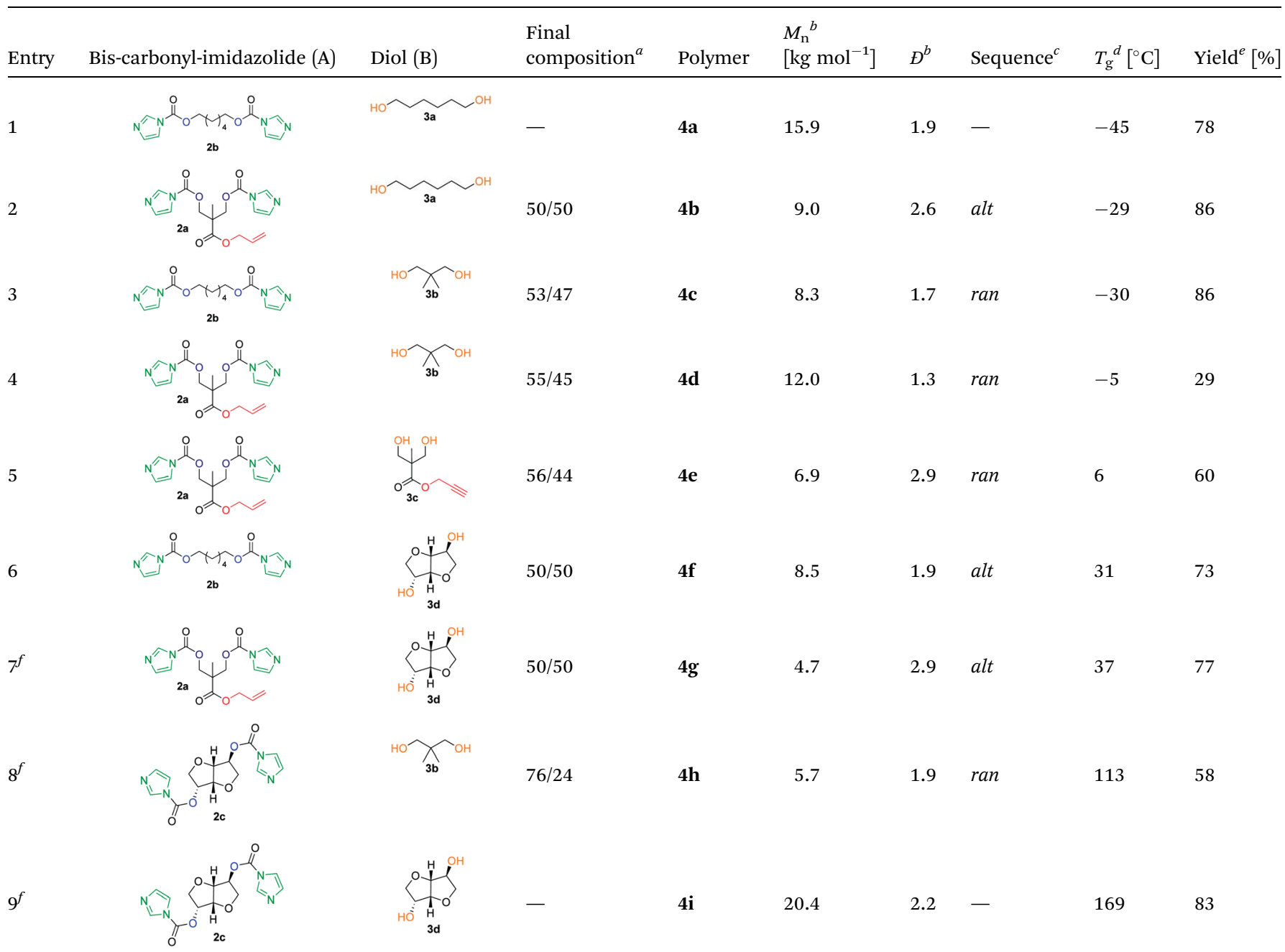

${ }^{a}$ All polymerizations were performed in sealed vials under argon atmosphere using a feed monomer ratio of $[\mathrm{A}]_{0} /[\mathrm{B}]_{0}=1.05: 1.00,5 \mathrm{~mol} \% \mathrm{CsF}$ as catalyst and EtOAc as solvent if not otherwise stated. ${ }^{b}$ Measured by SEC, theoretical $M_{\mathrm{n}}=5.9 \mathrm{~kg} \mathrm{~mol}^{-1}$ based on feed composition according to basic principles of step growth polymerization. ${ }^{23}{ }^{c}$ Determined by carbonyl dyad analysis ${ }^{13} \mathrm{C}-\mathrm{NMR}$ (Fig. 2 and S17-S19). ${ }^{d}$ Obtained from DSC on the second heating scan. ${ }^{e}$ Isolated yield after precipitation. ${ }^{f}$ Acetone was used as reaction solvent. 
Kinetic plots of $\ln \left([\mathrm{OH}]_{0} /[\mathrm{OH}]\right)$ vs. time showed a good fit to a first order reaction in $[\mathrm{OH}]$ when using an excess of diol 3a in the feed (Fig. 1B), and a progressive deviation towards second order behavior when increasing the bis-carbonylimidazolide $\mathbf{2 b}$ loading up to $5 \%$ molar excess (Fig. S1B $\dagger$ ). This is in agreement with previous findings for polyester dendrimer synthesis, ${ }^{22}$ where the strong H-bonding of fluoride anions greatly enhanced the nucleophilicity of hydroxyl groups, without inhibition from the protic imidazole leaving group, being a poorer electron acceptor. Investigation of the versatility of FPC to generate novel APCs was carried out using the selected conditions on a range of structurally and functionally relevant diols 3a-d, (Scheme 1, Table 1) displaying a library of variable spacer lengths, bulkiness and functionalities. In all cases, polycarbonates were obtained with molecular weights $\left(M_{\mathrm{n}}\right) 5-20 \mathrm{~kg}$ $\mathrm{mol}^{-1}$ and dispersities from 1.3 to 2.9 (Table 1 ). The homopolymers $4 \mathbf{a}$ and $4 \mathbf{i}$ displayed highest $M_{\mathrm{n}}$ of 16 and $20 \mathrm{~kg} \mathrm{~mol}^{-1}$ respectively (entries 1 and 10). In contrast to 1,6-diols, their 1,3counterparts are known to be more challenging substrates in step-growth polymerization due to competing ring-closing depolymerization processes. $^{24}$ Interestingly, FPC polymerization with strictly 1,3-configurations afforded the polycarbonate 4d (Table 1, entry 4 ) with relatively high $M_{\mathrm{n}}$, indicating that the step-growth reaction is favored over depolymerization. This may be attributed to the beneficial features of imidazole including its low nucleophilicity and distinct neutral character, enabling effective formation of polycarbonates without the need for continuous removal of the imidazole by-product. Also, more advanced APCs were accomplished by FPC of allyl-functional biscarbonylimidazolide monomer 2a with propargyl-functional 1,3diol 3c (entry 5). This enabled the incorporation of pendant orthogonal functionalities along the main chain well known to undergo chemoselective post-functionalization processes via thiol-ene ${ }^{25}$ and CuAAC $^{26}$ click chemistry. Additionally, the robustness of FPC was demonstrated by the introduction of bulky diols such as isosorbide 3d for the synthesis of APC $\mathbf{4 f}-\mathbf{i}$ with molecular weights ranging between $5-20 \mathrm{~kg} \mathrm{~mol}^{-1}$. All polymers were purified by simple precipitation into a methanol solution and thoroughly characterized using ${ }^{1} \mathrm{H},{ }^{13} \mathrm{C}-\mathrm{NMR}$ as well as SEC techniques (Fig. S6-S14 and S21†).

Analysis of carbonyl dyad shifts from ${ }^{13} \mathrm{C}-\mathrm{NMR}$ (Fig. 2A and $\mathrm{S} 17-\mathrm{S} 19 \dagger)$ for the APC synthesized via FPC indicated that homopolymers $\mathbf{4 b}$ (Table 1 , entry 2) and $\mathbf{4 f}-\mathbf{g}$ (entries 6-7) were alternating $(\mathrm{AB})$, while $\mathbf{4 d}-\mathbf{e}$ and $\mathbf{4 h}$ (entries 4-5 and 8) also displayed significant amounts of homo-coupled (AA and BB) sequences (see ESI for assignments $\dagger$ ). These results could be explained by a suggested mechanism for FPC (Fig. 2B), which elaborates on the assumption that the 1,3-diol based polycarbonates linkages may undergo sequence scrambling by reversible ring-closing depolymerization. Consequently, the mechanism is envisioned to involve two parallel mechanistic cycles, a main traditional step-growth and a chain-growth ROP-like mechanism. This was corroborated from the observed deviation in final monomer composition when using neopentyl glycol $\mathbf{3 b}$ (4c-d and $\mathbf{4 h}$, Table 1 ). The most pronounced deviation was observed for $\mathbf{4 h}$ which exhibited the characteristic ${ }^{1} \mathrm{H}-\mathrm{NMR}$ shifts of the cyclic carbonate analogue of $3 \mathrm{~b}$, at $4.07 \mathrm{ppm}$ $\left(\mathrm{s}, 4 \mathrm{H},-\mathrm{CH}_{2} \mathrm{O}\right)$ and $1.12 \mathrm{ppm}\left(\mathrm{s}, 6 \mathrm{H},-\mathrm{CH}_{3}\right),{ }^{27}$ during polymerization (Fig. S20 $\dagger$ ). This may be explained by a process involving partial ring-closing depolymerization of 1,3-diol chainends into its six-membered cyclic form, which would explain the high glass transition temperature $\left(T_{\mathrm{g}}\right)$ of $113{ }^{\circ} \mathrm{C}$ observed for $\mathbf{4 h}$ (entry 8). Interestingly, this pathway is effectively blocked when higher diol linkage (3a and 3d) segments are present, thus allowing formation of purely alternating polycarbonates such as $\mathbf{4 b}$ and 4f-g (Table 1, Fig. 2).

From a commercial point-of-view, a relevant advantage of FPC over ROP is that it allows the use of diol co-monomers with highly variable backbones. This was manifested by an efficient tuning of the thermal properties, showing a single $T_{\mathrm{g}}$ value for each APC ranging from -45 up to $169{ }^{\circ} \mathrm{C}$ (Table 1 and Fig. S22 $\dagger$ ). In particular, examples of high $T_{\mathrm{g}}$ polycarbonates obtained from ROP are limited, ${ }^{3,4}$ except for the recent report by Wooley and co-workers on the polymerization of a glucose-derived cyclic carbonate. ${ }^{28}$ The isosorbide (3d) has attracted considerable attention as a potential BPA replacement building block in renewable polymers. This is mainly due to the unique material properties of the polymers including high $T_{\mathrm{g}}$, great mechanical and optical properties. ${ }^{29}$ The polyisosorbide carbonate has recently entered the commercial market (DurabioTM) as a structural material in car interiors (by Mitsubishi cars) and smartphone screens (by Sharp technologies). However, it is still commercially produced by traditional step-growth protocols involving phosgene or DPC. In this context, the versatile nature

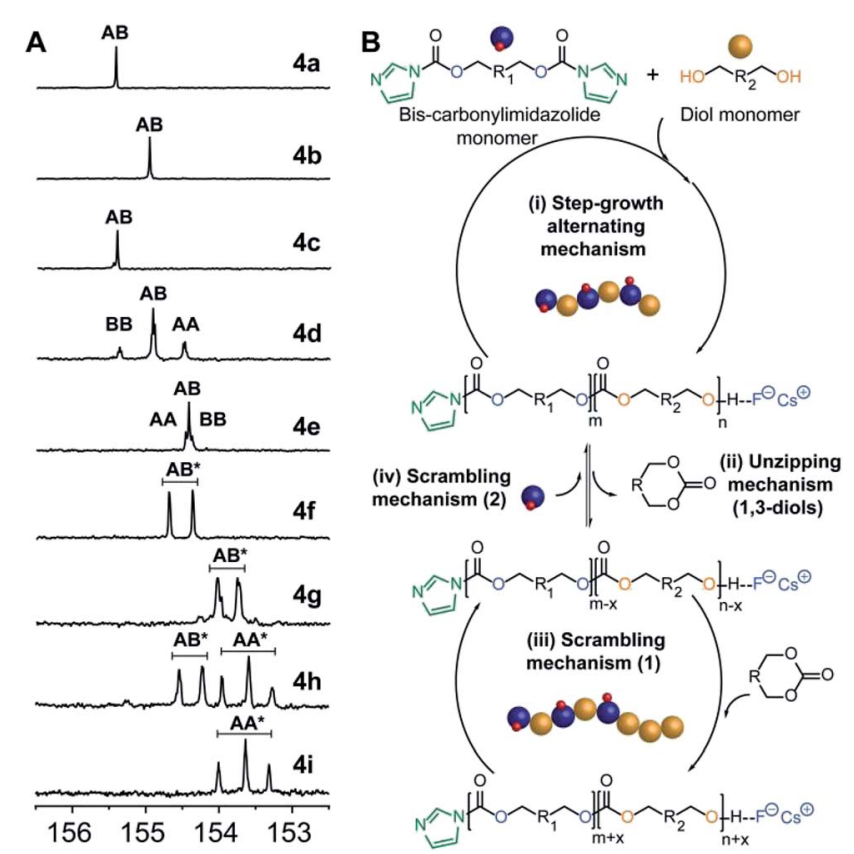

Fig. 2 (A) ${ }^{13} \mathrm{C}-\mathrm{NMR}$ carbonyl region of $4 \mathrm{a}-\mathrm{i}$, [*] refers to the multiple dyads resulting from the endo and exo hydroxyl configuration of isosorbide $4 \mathrm{f}-\mathrm{i}$, Fig. S18 and S19. $\dagger$ (B) Proposed simplified mechanistic cycles involving: (i) step-growth polymerization to afford alternating polycarbonates; (ii) unzipping mechanism involving ring-closing of hydroxyl terminal 1,3-units into six-membered cyclic carbonates; (iii) scrambling mechanism 1 involving ROP of cyclic carbonates to form BB dyads; and (iv) scrambling mechanism 2 involving bis-carbonylimidazolide monomer to form AA dyads. 
of FPC allowed the facile production of isosorbide based polymers, both the homopolymer $4 \mathrm{i}$ with $M_{\mathrm{n}}$ of $20 \mathrm{~kg} \mathrm{~mol}^{-1}$ and $T_{\mathrm{g}}$ of $169{ }^{\circ} \mathrm{C}$ (entry 9 ) as well as its allyl functional counterpart $\mathbf{4 f}$, obtained from polymerization of isosorbide $\mathbf{3 d}$ with $2 \mathbf{a}$ (entry 7 ). The polymer $\mathbf{4 f}$ is unique due to its perfect alternating configuration, high concentration of pendant allyl groups and relatively high $T_{\mathrm{g}}$ of $37^{\circ} \mathrm{C}$.

\section{Conclusions}

In conclusion, we have presented a facile and versatile two-step synthetic strategy to polycarbonates that exploit CsF as an efficient and novel inorganic catalyst acting under benign and mild reaction conditions. The FPC polymerization follows a traditional step-growth mechanism which is favoured over depolymerization reactions even for the challenging 1,3-diols. Nevertheless, these 1,3-diols can seemingly undergo an unzipping mechanism that leads to scrambled sequences in the polymer, unlike higher diols that produce purely alternating polycarbonates. The FPC polymerization methodology should serve as a complement to existing methodologies by combining the operational benefits of ROP and scalability of traditional step-growth polymerization protocols. The methodology allows fabrication of a wider range of previously inaccessible functional polycarbonate-based materials with structurally diverse backbones. We truly believe FPC will play a crucial role in the future availability of advanced polycarbonates and their use as materials in technical and biomedical engineering sectors.

\section{Acknowledgements}

This work was generously supported by the Swedish Research Council VR (2011-5358 and 2010-435) and Knut and Alice Wallenberg Foundation KAW (2012-0196). Vilhelm Olsson thanks Marcus and Amalia Wallenberg foundation (MAW). This project has received funding for Sandra García-Gallego from the European Union's Horizon 2020 research and innovation programme under the Marie Skłodowska-Curie Grant Agreement No. 655649.

\section{Notes and references}

1 D. G. LeGrand and J. T. Bendler, Handbook of Polycarbonate Science and Technology, Marcel Dekker, 2000.

2 E. Testai, P. Hartemann, E. Rodriguez-Farre, S. C. Rastogi, J. Bustos, U. Gundert-Remy, A. Hensten, H. M. Kopperud, N. Olea, A. Piersma and W. De Jong, Regul. Toxicol. Pharmacol., 2016, 79, 106-107.

3 J. Feng, R.-X. Zhuo and X.-Z. Zhang, Prog. Polym. Sci., 2012, 37, 211-236.

4 S. Tempelaar, L. Mespouille, O. Coulembier, P. Dubois and A. P. Dove, Chem. Soc. Rev., 2013, 42, 1312-1336.
5 J. Xu, E. Feng and J. Song, J. Appl. Polym. Sci., 2014, 131, 39822.

6 N. E. Kamber, W. Jeong, R. M. Waymouth, R. C. Pratt, B. G. G. Lohmeijer and J. L. Hedrick, Chem. Rev., 2007, 107, 5813-5840.

7 G. L. Gregory, M. Ulmann and A. Buchard, RSC Adv., 2015, 5, 39404-39408.

8 D. P. Sanders, K. Fukushima, D. J. Coady, A. Nelson, M. Fujiwara, M. Yasumoto and J. L. Hedrick, J. Am. Chem. Soc., 2010, 132, 14724-14726.

9 J. V. Olsson, D. Hult, Y. Cai, S. Garcia-Gallego and M. Malkoch, Polym. Chem., 2014, 5, 6651-6655.

10 P. Olsén, K. Odelius and A.-C. Albertsson, Macromolecules, 2014, 47, 6189-6195.

11 D. J. Darensbourg, R. M. Mackiewicz, A. L. Phelps and D. R. Billodeaux, Acc. Chem. Res., 2004, 37, 836-844.

12 X.-B. Lu, W.-M. Ren and G.-P. Wu, Acc. Chem. Res., 2012, 45, 1721-1735.

13 R. Paul and G. W. Anderson, J. Am. Chem. Soc., 1960, 82, 4596-4600.

14 M. J. Ford and S. V. Ley, Synlett, 1990, 1990, 255-256.

15 S. P. Rannard and N. J. Davis, Org. Lett., 1999, 1, 933-936.

16 W. J. Feast, S. P. Rannard and A. Stoddart, Macromolecules, 2003, 36, 9704-9706.

17 A. Stoddart, W. J. Feast and S. P. Rannard, Soft Matter, 2012, 8, 1096-1108.

18 S. P. Rannard and N. J. Davis, J. Am. Chem. Soc., 2000, 122, 11729-11730.

19 D. J. Dale, P. J. Dunn, C. Golightly, M. L. Hughes, P. C. Levett, A. K. Pearce, P. M. Searle, G. Ward and A. S. Wood, Org. Process Res. Dev., 2000, 4, 17-22.

20 J. M. J. Fréchet, F. M. Houlihan, F. Bouchard, B. Kryczka and C. G. Wilson, J. Chem. Soc., Chem. Commun., 1985, 15141516.

21 K. Alfonsi, J. Colberg, P. J. Dunn, T. Fevig, S. Jennings, T. A. Johnson, H. P. Kleine, C. Knight, M. A. Nagy, D. A. Perry and M. Stefaniak, Green Chem., 2008, 10, 31-36.

22 S. Garcia-Gallego, D. Hult, J. V. Olsson and M. Malkoch, Angew. Chem., Int. Ed., 2015, 54, 2416-2419.

23 P. J. Flory, Principles of Polymer Chemistry, Cornell Univ. Press, 1953.

24 W. H. Carothers and F. J. Van Natta, J. Am. Chem. Soc., 1930, 52, 314-326.

25 C. E. Hoyle and C. N. Bowman, Angew. Chem., Int. Ed., 2010, 49, 1540-1573.

26 J.-F. Lutz, Angew. Chem., Int. Ed., 2007, 46, 1018-1025.

27 P. Loewenhielm, H. Claesson and A. Hult, Macromol. Chem. Phys., 2004, 205, 1489-1496.

28 K. Mikami, A. T. Lonnecker, T. P. Gustafson, N. F. Zinnel, P.-J. Pai, D. H. Russell and K. L. Wooley, J. Am. Chem. Soc., 2013, 135, 6826-6829.

29 M. Rose and R. Palkovits, ChemSusChem, 2012, 5, 167-176. 